\title{
Peripheral mononuclear blood cells contribute to the obesity- associated inflammatory state independently of glycemic status: involvement of the novel proinflammatory adipokines chemerin, chitinase-3-like protein 1, lipocalin-2 and osteopontin
}

\author{
Victoria Catalán ${ }^{1,2,3} \cdot$ Javier Gómez-Ambrosi ${ }^{1,2,3} \cdot$ Amaia Rodríguez $^{1,2,3}$. \\ Beatriz Ramírez ${ }^{1,2,3} \cdot$ Víctor Valenti ${ }^{2,4}$ • Rafael Moncada ${ }^{3,5}$ • Camilo Silva ${ }^{2,3,6}$. \\ Javier Salvador $^{2,6} \cdot$ Gema Frühbeck ${ }^{1,2,3,6}$
}

Received: 24 December 2014/ Accepted: 28 March 2015/Published online: 14 April 2015

(C) Springer-Verlag Berlin Heidelberg 2015

\begin{abstract}
Inflammation is a critical contributor to the pathogenesis of metabolic disorders with adipose tissue being crucial in the inflammatory response by releasing multiple adipokines with either pro- or anti-inflammatory activities with potential functions as metabolic regulators. Peripheral blood mononuclear cells (PBMC) have been proposed as representative of the inflammatory status in obesity. The aim of the present study was to evaluate the contribution of PBMC to the obesity-associated chronic inflammation analyzing the expression of novel adipokines. Samples obtained from 69 subjects were used in the study. Real-time PCR determinations were performed to quantify gene expression levels in PBMC of novel adipokines
\end{abstract}

Electronic supplementary material The online version of this article (doi:10.1007/s12263-015-0460-8) contains supplementary material, which is available to authorized users.

Victoria Catalán

vcatalan@unav.es

Gema Frühbeck

gfruhbeck@unav.es

1 Metabolic Research Laboratory, Clínica Universidad de Navarra, Pamplona, Spain

2 CIBER Fisiopatología de la Obesidad y Nutrición (CIBERobn), Instituto de Salud Carlos III, Pamplona, Spain

3 Obesity and Adipobiology Group, Instituto de Investigación Sanitaria de Navarra (IdiSNA), Pamplona, Spain

4 Department of Surgery, Clínica Universidad de Navarra, Pamplona, Spain

5 Department of Anesthesia, Clínica Universidad de Navarra, Pamplona, Spain

6 Department of Endocrinology and Nutrition, Clínica Universidad de Navarra, Avda. Pío XII, 36, 31008 Pamplona, Spain including chemerin, chitinase-3-like protein 1 (YKL-40), lipocalin-2 (LCN-2) and osteopontin (OPN), and their circulating concentrations were also determined by ELISA. We show, for the first time, that PBMC gene expression levels of chemerin $(P<0.0001)$, chitinase-3-like protein 1 $(P=0.010)$, lipocalin-2 $(P<0.0001)$ and osteopontin $(P<0.0001)$ were strongly upregulated in obesity independently of the glycemic state. Circulating concentrations of these adipokines followed the same trend being significantly higher $(P<0.05)$ in obese normoglycemic and type 2 diabetic patients compared to lean volunteers and also associated $(P<0.05)$ with their corresponding mRNA levels in PBMC. These results provide evidence that alterations in inflammation-related adipokines are manifest in PBMC, which might contribute to the low-grade chronic inflammation that characterizes obesity.

Keywords Inflammation - Obesity $\cdot$ Peripheral blood cells $\cdot$ Adipokines

\section{Introduction}

Obesity is a worldwide health problem associated with several comorbidities including hypertension, dyslipidemia, type 2 diabetes mellitus (T2D), coronary heart disease, stroke, osteoarthritis, sleep apnea and respiratory problems, as well as some types of cancers (Campo et al. 2007; Frühbeck 2012; Frühbeck et al. 2013; Ng et al. 2014). Obesity has profound effects on immunity and inflammation being critical contributors to the pathogenesis of these metabolic disorders (Esser et al. 2014; Klöting and Blüher 2014; Lumeng and Saltiel 2011). In this sense, white blood cell counts (Dixon and O'Brien 2006; Vozarova et al. 2002) as well as plasma levels of acute- 
phase proteins (Gómez-Ambrosi et al. 2006; Yudkin et al. 1999) and pro-inflammatory cytokines (Catalán et al. 2007a; Lasselin et al. 2014) are elevated in obese and T2D patients experimenting a reduction after weight loss (Bastard et al. 2000; Catalán et al. 2011). The obesity-associated low-grade inflammation results in the persistent stimulation of the immune system mainly characterized by the infiltration of adipose tissue with macrophages (Poulain-Godefroy et al. 2008; Weisberg et al. 2003) and the activation of circulating immune cells shifting toward a pro-inflammatory profile (Ghanim et al. 2004; Lumeng et al. 2007).

Different studies have suggested that the gene expression profile of peripheral blood mononuclear cells (PBMC) reflects the visceral fat amount and may be representative of the inflammatory status in obesity (Ghanim et al. 2004; Yamaoka et al. 2012, 2013) although they are not necessarily the primary source for elevated circulating inflammatory cytokines in obesity (O'Rourke et al. 2006). PBMC from obese individuals showed an increased secretion of the pro-inflammatory cytokines tumor necrosis factor (TNF)- $\alpha$, interferon (IFN)- $\gamma$ and interleukin (IL)-2 as well as a lower production of the anti-inflammatory cytokine IL10 (Dicker et al. 2013). Reportedly, T2D obese patients exhibit higher expression levels of TNF receptor (TNFR)-2 in PBMC compared to normoglycemic obese individuals (Fogeda et al. 2004). Moreover, diet-induced weight loss in individuals with metabolic syndrome altered the gene expression of cytokines related to inflammation (de Mello et al. 2008b) and also resulted in a decreased expression of genes involved in the activation of the transcription factor nuclear factor $\kappa \mathrm{B}(\mathrm{NF \kappa B})$ in PBMC (de Mello et al. 2008a). In addition, an association between white blood cell subtypes and measures of insulin resistance has been recently described (Lee et al. 2014). Importantly, obese patients exhibit reduced immune regulatory $\mathrm{T}$ (Tregs) cells (Wagner et al. 2013), and crucial defense against inappropriate immune responses operating in contexts of inflammation (Feuerer et al. 2009; Sakaguchi et al. 2008).

In recent years, new factors secreted by adipose tissue that promote inflammatory responses and metabolic dysfunction or contribute to the resolution of inflammation have been identified (Olefsky and Glass 2010). The balance between these factors is crucial for determining homeostasis (Frühbeck and Gómez-Ambrosi 2003; Leal Vde and Mafra 2013; Ouchi et al. 2011). In states of obesity, we have previously described that adipose tissue produces higher levels of novel adipokines including chemerin, chitinase-3-like protein 1 (YKL-40), lipocalin-2 (LCN-2) and osteopontin (OPN) (Catalán et al. 2009, 2011, 2013; Gómez-Ambrosi et al. 2007). The family of adipokines is highly diverse in structure and function. Whereas LCN-2 seems to act as an antagonist to the effect of inflammatory molecules (Guo et al. 2014; Zhang et al. 2008), OPN and YKL-40 are directly related to increased inflammation and insulin resistance via impaired extracellular matrix remodeling (Johansen et al. 2000; Lancha et al. 2014).

Since previous studies have mainly focused on the analysis of mediators of inflammation in adipose tissue, muscle or liver, we aim to evaluate whether alterations in the gene expression levels of novel adipokines whose regulation is altered in adipose tissue in obesity are equally manifested in PBMC, thereby providing to be useful circulating biomarkers.

\section{Materials and methods}

\section{Patient selection}

In order to analyze the effect of obesity and T2D on the circulating and gene expression levels of novel inflammation-related adipokines, blood samples from 69 subjects [20 normoglycemic lean (LN), 24 normoglycemic obese (OB-NG) and 25 obese with T2D (OB-T2D)] recruited from healthy volunteers and patients attending the Departments of Endocrinology and Nutrition as well as Surgery at the Clínica Universidad de Navarra were used. Patients underwent a clinical assessment including medical history, physical examination, body composition analysis and comorbidity evaluation performed by a multidisciplinary consultation team. Body mass index (BMI) was calculated as weight in kilograms divided by the square of height in meters and body fat (BF) was estimated by air displacement plethysmography (Bod-Pod ${ }^{\circledR}$, Life Measurements, Concord, CA) (Gómez-Ambrosi et al. 2012). Patients were classified as obese according to BMI $\geq 30 \mathrm{~kg} /$ $\mathrm{m}^{2}$ as well as $\mathrm{BF} \geq 25 \%$ for males and $\mathrm{BF} \geq 35 \%$ for females. The waist-to-hip ratio (WHR) was measured as the quotient between the circumference of the waist (at the midway level between the margin of the lowest rib and the iliac crest) and the hip (at the widest trochanters). Normoglycemia and T2D were defined following the criteria of the Expert Committee on the Diagnosis and Classification of Diabetes Mellitus (Diagnosis and classification of diabetes mellitus 2014). T2D of obese patients was of recent diagnosis, and the patients were not on insulin therapy or on medication likely to influence endogenous insulin levels. Patients with chronic diseases, other than obesity, such as cancer pathology or infectious diseases were excluded from the study. The study was approved, from an ethical and scientific standpoint, by the Hospital's Ethical Committee responsible for research, and the written informed consent of participants was obtained. 


\section{Laboratory procedures}

Plasma samples were obtained by venipuncture after an overnight fast. Glucose was analyzed based on enzymatic spectrophotometric reactions by an automated analyzer (Hitachi Modular P800, Roche, Basel, Switzerland). Insulin was measured by means of an enzyme-amplified chemiluminescence assay (IMMULITE ${ }^{\circledR}$, Diagnostic Products Corp., Los Angeles, CA) with intra- and interassay coefficients of variation of 4.2 and $5.7 \%$, respectively. Insulin resistance and sensitivity were calculated using the HOMA and QUICKI indices, respectively. Total cholesterol, high-density lipoprotein (HDL-cholesterol) and lowdensity lipoprotein (LDL-cholesterol) levels were calculated as previously described (Catalán et al. 2007a). Alanine aminotransferase (ALT), aspartate aminotransferase (AST), alkaline phosphatase (ALP), $\gamma$-glutamyltransferase $(\gamma-\mathrm{GT})$ and creatinine were measured by enzymatic tests (Roche) in an automated analyzer (Roche/Hitachi Modular P800). High-sensitivity C-reactive protein (CRP), fibrinogen, homocysteine and von Willebrand factor antigen (vWF) concentrations were determined as previously reported (Catalán et al. 2007a). Leptin was measured by a double-antibody RIA method (Linco Research, Inc., St. Charles, MO); intra- and inter-assay coefficients of variation were 5.0 and $4.5 \%$, respectively. Commercially available ELISA kits were used to assess circulating levels of LCN-2 (R \& D systems, Minneapolis, MN), OPN (R \& D systems), YKL-40 (R \& D systems) and chemerin (Biovendor, Heidelberg, Germany) according to the manufacturer's instructions. The intra- and interassay coefficients of variation were as follows: 3.7 and $6.5 \%$ for LCN-2; 3.2 and $5.9 \%$ for OPN; 4.6 and $6.0 \%$ for YKL40 ; and 6.0 and $7.6 \%$ for chemerin.

\section{RNA extraction and Real-Time PCR}

Human peripheral blood mononuclear cells were isolated by density gradient centrifugation on Ficoll (GE Healthcare Life Sciences, Pittsburgh, PA), and RNA extraction was performed using the RNeasy Tissue Mini Kit (Qiagen, Valencia, CA) according to the manufacturer's directions and treated with DNase I (RNase-free DNase Set, Qiagen) in order to remove any trace of genomic DNA. For firststrand cDNA synthesis, constant amounts of $1 \mu \mathrm{g}$ of total RNA were reverse-transcribed in a $20-\mu \mathrm{L}$ final volume using random hexamers (Roche) as primers and 200 units of M-MLV reverse transcriptase (Invitrogen, Carlsbad, CA) (Gómez-Ambrosi et al. 2004).

The transcript levels for LCN-2 (LCN2), OPN (SPP1), YKL-40 (CHI3L1) and chemerin (RARRES2) were quantified by Real-Time PCR (7300 Real-Time PCR System, Applied Biosystem, Foster City, CA). Primers and probes
(Supplemental Table 1) were designed using the software Primer Express 2.0 (Applied Biosystems) and purchased from Genosys (Sigma-Aldrich, Madrid, Spain). Primers or TaqMan ${ }^{\circledR}$ probes encompassing fragments of the areas from the extremes of two exons were designed to ensure the detection of the corresponding transcript avoiding genomic DNA amplification. The cDNA was amplified at the following conditions: $95^{\circ} \mathrm{C}$ for $10 \mathrm{~min}$, followed by 45 cycles of $15 \mathrm{~s}$ at $95{ }^{\circ} \mathrm{C}$ and $1 \mathrm{~min}$ at $59{ }^{\circ} \mathrm{C}$, using the TaqMan ${ }^{\circledR}$ Universal PCR Master Mix (Applied Biosystems). The primer and probe concentrations for gene amplification were 300 and $200 \mathrm{nmol} / \mathrm{L}$, respectively. All results were normalized to the levels of the ribosomal $18 \mathrm{~S}$ rRNA (Applied Biosystems), and relative quantification was calculated using the $\Delta \Delta \mathrm{Ct}$ formula. Relative mRNA expression was expressed as fold expression over the calibrator sample (average of gene expression corresponding to the LN group) as previously described (Catalán et al. 2007b). All samples were run in triplicate, and the average values were calculated. All PCR amplification efficiencies were close to $100 \%$, indicating that the amount of product doubles with each cycle (Bustin et al. 2009) (Supplemental Fig. 1).

\section{Statistical analysis}

Data are presented as mean \pm standard error of the mean (SEM). The program PS Power and Sample Size Calculations (edition 3.0.43) was used to determine the power of the study and sample size calculation. In order to identify biologically significant differences between groups, we performed an estimation of a continuous response variable (plasma OPN concentrations). Based on previous similar studies of our own group, the standard deviation was expected to be $16 \mathrm{ng} / \mathrm{mL}$, thereby yielding the need to study at least 18 patients to be able to reject the null hypothesis with a 0.9 power expecting a minimum mean change of $18 \mathrm{ng} / \mathrm{mL}$ in an unpaired comparison between two groups. The type I error probability associated was 0.05 . Anticipating a potential loss of samples due to sample availability or methodological issues, we decided to use at least 20 patients per group. Due to the good attendance of patients to our departments, the final experimental design included 20, 24 and 25 patients in the lean, obese normoglycemic and obese with type 2 diabetes groups, respectively. Differences in the proportion of subjects within groups regarding gender were assessed by Chi-square test. Due to their non-normal distribution, CRP concentrations as well as gene expression levels of the inflammatory markers were logarithmically transformed. The normal distribution of the other variables was adequate for the use of parametric tests. Differences in the proportion of subjects within groups 
regarding gender were assessed by using a contingency test (Chi-square test). Differences between groups were assessed by one-way ANOVA followed by Tukey's post hoc tests and were further analyzed after age adjustment by analysis of covariance (ANCOVA). Associations between two variables were computed by Pearson's $(r)$ correlation coefficient. The calculations were performed using the SPSS/Windows version 15.0 statistical package (SPSS, Chicago, IL, USA). A $P$ value $<0.05$ was considered statistically significant.

\section{Results}

\section{Patients' characteristics}

The biochemical and hormonal characteristics of the subjects included in the study are shown in Table 1. No differences in gender distribution between groups were found $(P=0.554)$. The obese patients exhibited significantly higher $(P<0.01)$ BMI, BF and waist circumference compared to the lean volunteers. As expected, obese
Table 1 Anthropometric and biochemical characteristics of subjects included in the study

\begin{tabular}{|c|c|c|c|c|}
\hline & Lean & Obese NG & Obese T2D & $P$ \\
\hline$n$ (male, female) & $20(8,12)$ & $24(7,17)$ & $25(8,17)$ & 0.554 \\
\hline Age (years) & $31 \pm 2$ & $35 \pm 3$ & $46 \pm 2^{*},^{\dagger}$ & $<0.01$ \\
\hline BMI $\left(\mathrm{kg} / \mathrm{m}^{2}\right)$ & $21.3 \pm 0.5$ & $43.4 \pm 1.2 * * *$ & $44.2 \pm 1.5 * * *$ & $<0.001$ \\
\hline Body fat (\%) & $22.3 \pm 1.5$ & $53.0 \pm 1.1 * * *$ & $50.5 \pm 1.3 * * *$ & $<0.001$ \\
\hline Waist $(\mathrm{cm})$ & $76 \pm 3$ & $122 \pm 3^{* * *}$ & $125 \pm 2 * * *$ & $<0.001$ \\
\hline Hip (cm) & $94 \pm 1$ & $132 \pm 2 * * *$ & $133 \pm 3 * * *$ & $<0.001$ \\
\hline Waist-to-hip ratio & $0.81 \pm 0.02$ & $0.93 \pm 0.2 * * *$ & $0.94 \pm 0.01 * * *$ & $<0.001$ \\
\hline SBP (mmHg) & $107 \pm 3$ & $124 \pm 3 * * *$ & $133 \pm 3^{* * *}$ & $<0.001$ \\
\hline DBP (mmHg) & $66 \pm 2$ & $76 \pm 2^{*}$ & $83 \pm 2 * * *, \dagger$ & $<0.001$ \\
\hline Fasting glucose $(\mathrm{mg} / \mathrm{dL})$ & $84 \pm 5$ & $85 \pm 2$ & $122 \pm 8 * * *, \dagger \dagger$ & $<0.001$ \\
\hline 2 h OGTT glucose $(\mathrm{mg} / \mathrm{dL})$ & - & $111 \pm 5$ & $168 \pm 10^{\dagger \dagger \dagger}$ & \\
\hline Fasting insulin $(\mu \mathrm{U} / \mathrm{mL})$ & $5.9 \pm 1.3$ & $15.1 \pm 1.6^{*}$ & $21.7 \pm 1.8^{* * *, \dagger}$ & $<0.001$ \\
\hline $2 \mathrm{~h}$ OGTT insulin $(\mu \mathrm{U} / \mathrm{mL})$ & - & $80.9 \pm 10.5$ & $136.4 \pm 15.4^{\dagger \dagger \dagger}$ & \\
\hline HOMA & $1.3 \pm 0.4$ & $3.1 \pm 0.3$ & $5.6 \pm 0.5^{* * *, \dagger \dagger}$ & $<0.001$ \\
\hline QUICKI & $0.386 \pm 0.015$ & $0.330 \pm 0.006 * * *$ & $0.303 \pm 0.004 * * *, \dagger$ & $<0.001$ \\
\hline Triacylglycerol (mg/dL) & $67 \pm 9$ & $100 \pm 9$ & $195 \pm 39 *{ }^{\dagger}$ & 0.015 \\
\hline Total cholesterol (mg/dL) & $176 \pm 9$ & $183 \pm 9$ & $211 \pm 9$ & 0.033 \\
\hline LDL-cholesterol (mg/dL) & $86 \pm 6$ & $109 \pm 7$ & $129 \pm 8 * *$ & 0.008 \\
\hline HDL-cholesterol (mg/dL) & $80 \pm 10$ & $54 \pm 4 * *$ & $49 \pm 2 * *$ & $<0.01$ \\
\hline Leptin (ng/mL) & $8.6 \pm 1.7$ & $57.0 \pm 5.2 * * *$ & $52.2 \pm 5.7 * * *$ & $<0.001$ \\
\hline CRP (mg/L) & $0.6 \pm 0.1$ & $10.2 \pm 1.9 * *$ & $7.7 \pm 1.3^{*}$ & $<0.001$ \\
\hline Fibrinogen (mg/dL) & $189 \pm 5$ & $379 \pm 17 * * *$ & $349 \pm 16^{* * *}$ & $<0.001$ \\
\hline von Willebrand factor (\%) & $61 \pm 9$ & $127 \pm 11^{* * *}$ & $136 \pm 11 * * *$ & $<0.001$ \\
\hline Homocysteine $(\mu \mathrm{mol} / \mathrm{L})$ & $6.6 \pm 0.6$ & $8.8 \pm 0.8$ & $9.9 \pm 1.2$ & 0.178 \\
\hline AST (UI/L) & $13 \pm 1$ & $16 \pm 1$ & $16 \pm 2$ & 0.556 \\
\hline ALT (UI/L) & $6 \pm 1$ & $24 \pm 3^{*}$ & $27 \pm 3 * * *$ & 0.006 \\
\hline ALP (UI/L) & $86 \pm 8$ & $100 \pm 5$ & $100 \pm 6$ & 0.367 \\
\hline$\gamma$-GT (UI/L) & $12 \pm 2$ & $18 \pm 3$ & $37 \pm 10$ & 0.093 \\
\hline
\end{tabular}

Data are mean \pm SEM. CRP was logarithmically transformed for statistical analysis due to its non-normal distribution. Differences regarding gender were assessed by Chi-square test. Differences between groups were analyzed by one-way ANOVA followed by Tukey's post hoc tests. Statistical differences in OGTT tests were determined by Student's $t$ test

$A L P$ alkaline phosphatase, $A L T$ alanine aminotransferase, $A S T$ aspartate aminotransferase, $B M I$ body mass index, $C R P$ C-reactive protein, $D B P$ diastolic blood pressure; $\gamma$-GT $\gamma$-glutamyltransferase, HOMA homeostasis model assessment, $N G$ normoglycemic, OGTT oral glucose tolerance test, QUICKI quantitative insulin sensitivity check index, $S B P$ systolic blood pressure, $T 2 D$ type 2 diabetes

$* P<0.05, * * P<0.01$ and $* * * P<0.001$ versus lean
${ }^{\dagger} P<0.05,{ }^{\dagger} P<0.01$ and ${ }^{\dagger \dagger} P<0.001$ versus obese NG 
Fig. 1 Circulating

concentrations of a LCN-2,

b YKL-40, c chemerin and d OPN of lean (LN), obese normoglycemic (OB-NG) and obese diabetic (OB-T2D) volunteers. Bars represent the mean \pm SEM $(\mathrm{LN} n=20$; OBNG $n=24$; OB-T2D $n=25$ ). Differences between groups were analyzed by one-way ANOVA followed by Tukey's tests. $* P<0.05 ; * * P<0.01$ versus $\mathrm{LN}$
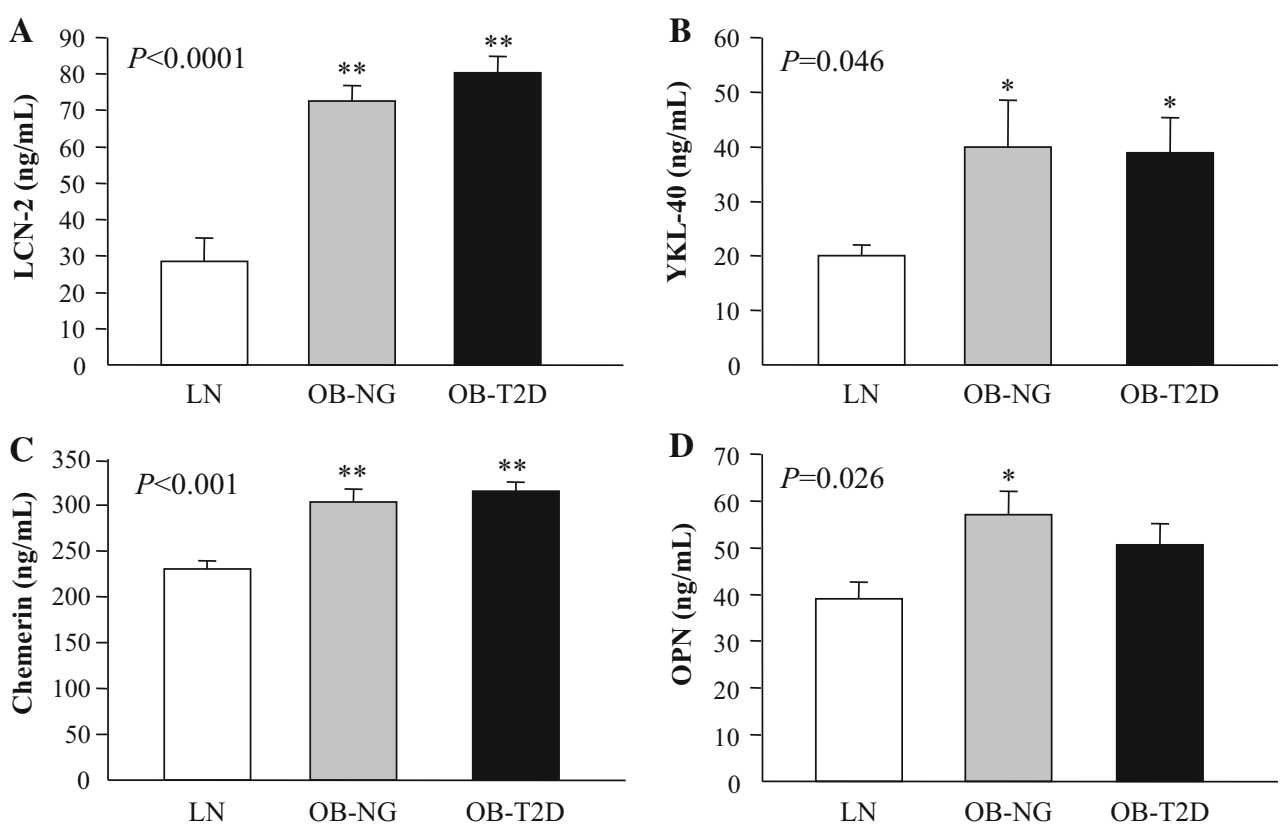

patients showed increased concentrations of leptin $(P<0.001)$, triacylglycerol $(P<0.001)$ and LDL-cholesterol $(P<0.05)$ levels together with lower $(P<0.05)$ circulating HDL-cholesterol concentrations. Obese patients with T2D exhibited higher HOMA $(P<0.01)$ and lower QUICKI $(P<0.01)$ indices than both lean and obese NG individuals. Circulating levels of the inflammatory markers CRP $\quad(P<0.05)$, fibrinogen $(P<0.01)$ and $\quad \mathrm{WWF}$ $(P<0.01)$ were increased in obese patients compared to lean subjects as well as the concentration of the hepatic enzyme $\operatorname{ALT}(P<0.01)$.

\section{Increased circulating levels of novel inflammatory adipokines in obesity}

Circulating concentrations of LCN-2 $(P<0.0001)$, YKL$40 \quad(P<0.05)$ and chemerin $(P<0.0001)$ were significantly increased in both obese groups compared to lean subjects (Fig. 1). OPN levels were significantly higher $(P<0.05)$ in obese NG patients, but no differences were found in obese subjects with T2D. Since obese patients with T2D were significantly older, an ANCOVA with age as a covariable was performed to investigate the effect of age on circulating levels of all the inflammatory-related adipokines. The differences in circulating concentrations of LCN-2 and chemerin due to obesity were also maintained after age adjustment $(P<0.001)$. Serum levels of OPN and YKL-40 in obese patients were increased, but differences were not statistically significant after age adjustment. In this line, circulating levels of LCN-2, YKL-40 and chemerin were positively correlated $(P<0.01)$ with different anthropometric measurements, but no association was detected regarding OPN levels (Table 2). LCN-2 $(P<0.001)$ and chemerin $(P<0.01)$ were highly positively associated with leptin and insulin levels as well as negatively correlated with the insulin sensitivity QUICKI index (Table 2). Importantly, a positive correlation $(P<0.01)$ between chemerin and LCN-2 with the classic inflammatory markers CRP, fibrinogen and vWF was detected (Table 2). Moreover, chemerin levels were also associated $(P<0.01)$ with all the new adipokines analyzed.

\section{Gene expression levels of inflammatory adipokines in peripheral mononuclear blood cells are increased in obese patients}

In light of the important contribution to obesity-associated inflammation of PBMC, we assessed gene expression levels of the inflammatory factors LCN2, CHI3L1, SPP1 and RARRES 2 in this type of cells. We detected an important upregulation $(P<0.01)$ of all the inflammatory adipokines in both groups of obese patients with similar results after age adjustment analysis (Fig. 2). Moreover, a high positive association $(P<0.001)$ between the gene expression levels of all the adipokines analyzed was found. As expected, a strong association between the mRNA levels of LCN2, CHI3L1, SPP1 and RARRES2 with anthropometric measurements was detected, whereas a negative association with the QUICKI index was found (Table 3). In line with the results found in the circulating levels, gene expression levels of LCN2 and SPP1 were 
Table 2 Univariate analysis of the correlations between circulating concentrations of the analyzed inflammatory markers and anthropometric and biochemical variables

\begin{tabular}{|c|c|c|c|c|c|c|c|c|}
\hline & \multicolumn{2}{|c|}{ Circulating LCN-2 } & \multicolumn{2}{|c|}{ Circulating YKL-40 } & \multicolumn{2}{|c|}{ Circulating OPN } & \multicolumn{2}{|c|}{ Circulating chemerin } \\
\hline & $r$ & $P$ & $r$ & $P$ & $r$ & $P$ & $r$ & $P$ \\
\hline Age & 0.33 & 0.007 & 0.35 & 0.006 & 0.02 & 0.836 & 0.47 & $<\mathbf{0 . 0 0 1}$ \\
\hline BMI & 0.70 & $<\mathbf{0 . 0 0 1}$ & 0.38 & 0.003 & 0.11 & 0.392 & 0.61 & $<\mathbf{0 . 0 0 1}$ \\
\hline Body fat & 0.76 & $<\mathbf{0 . 0 0 1}$ & 0.24 & 0.311 & 0.10 & 0.488 & 0.72 & $<\mathbf{0 . 0 0 1}$ \\
\hline Fasting glucose & 0.15 & 0.284 & 0.03 & 0.802 & 0.13 & 0.375 & 0.08 & 0.664 \\
\hline Fasting insulin & 0.42 & 0.002 & 0.02 & 0.870 & 0.07 & 0.633 & 0.41 & 0.016 \\
\hline QUICKI & -0.53 & $<\mathbf{0 . 0 0 1}$ & -0.09 & 0.532 & -0.01 & 0.910 & -0.57 & $<\mathbf{0 . 0 0 1}$ \\
\hline Leptin & 0.46 & $<\mathbf{0 . 0 0 1}$ & 0.19 & 0.201 & 0.04 & 0.785 & 0.50 & 0.003 \\
\hline CRP & 0.56 & $<\mathbf{0 . 0 0 1}$ & -0.05 & 0.731 & 0.20 & 0.201 & 0.47 & 0.009 \\
\hline Fibrinogen & 0.53 & $<\mathbf{0 . 0 0 1}$ & 0.08 & 0.568 & 0.236 & 0.119 & 0.46 & 0.006 \\
\hline von Willebrand factor & 0.33 & $\mathbf{0 . 0 2 3}$ & 0.21 & 0.173 & 0.26 & 0.096 & 0.58 & $<\mathbf{0 . 0 0 1}$ \\
\hline AST & 0.06 & 0.635 & 0.34 & 0.017 & 0.09 & 0.522 & 0.17 & 0.329 \\
\hline ALT & 0.28 & $\mathbf{0 . 0 3 9}$ & 0.32 & 0.025 & 0.17 & 0.252 & 0.33 & 0.054 \\
\hline
\end{tabular}

Bold values denote statistically significant $P$ values

$A L T$ alanine aminotransferase, $A S T$ aspartate aminotransferase, BMI body mass index, CRP C-reactive protein, $Q U I C K I$ quantitative insulin sensitivity check index

Fig. 2 Gene expression levels in peripheral blood mononuclear cells (PBMC) of a lipocalin-2 ( $L C N 2)$, b YKL-40 (CHI3L1), c chemerin (RARRES2) and d OPN (SPP1) of lean (LN), obese normoglycemic (OB-NG) and obese diabetic (OB-T2D) volunteers. The mRNA bars represent the mean \pm SEM of the ratio between the gene expression and $18 S$ rRNA. The expression level in the $\mathrm{LN}$ subjects was assumed to be 1 (LN, $n=20$; OB-NG, $n=24$; OB-T2D, $n=25$ ). Differences between groups were analyzed by one-way ANOVA followed by Tukey's tests. ${ }^{*} P<0.05$; $* * P<0.01$ versus $\mathrm{LN}$
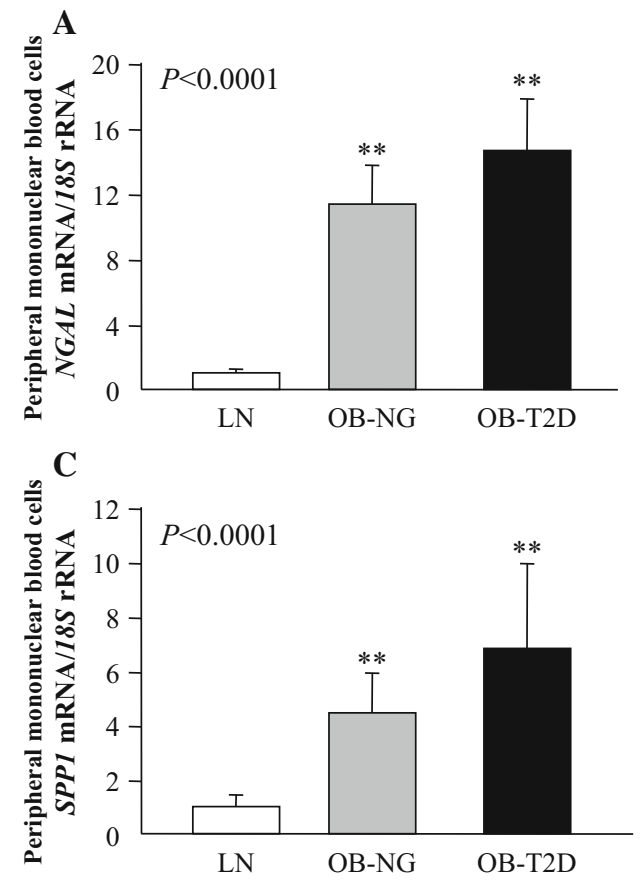
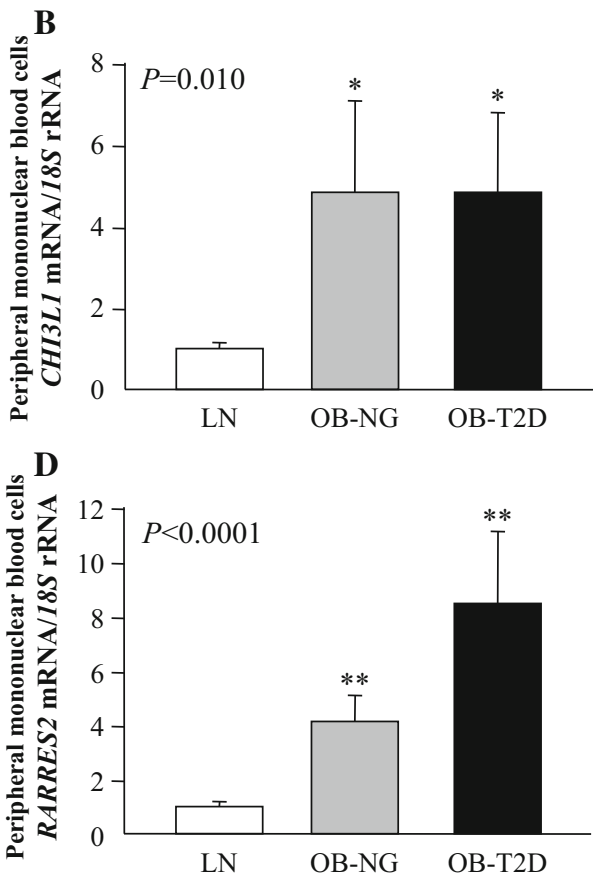

associated with classic inflammatory markers, detecting a positive association with circulating levels of CRP, fibrinogen and vWF (Table 3). Gene expression levels of all adipokines were associated with leptin concentrations except for CHI3L1 (Table 3).

Finally, we also identify a positive association between the gene expression levels of all adipokines analyzed with their corresponding circulating concentrations, except for OPN (Table 4).

\section{Discussion}

Different factors are involved in the obesity-related comorbidities but chronic low-grade inflammation is emerging as a dominant overarching view (Johnson and Olefsky 2013). During obesity, cells from the innate and adaptive immune system infiltrate insulin-sensitive tissues, starting the inflammatory response (Weisberg et al. 2003; Xu et al. 2003). Adipose tissue is an important initiator of the inflammatory 
Table 3 Univariate analysis of the correlations between gene expression levels of the analyzed inflammatory markers and anthropometric and biochemical variables
Table 4 Univariate analysis of the correlations between gene expression levels and circulating concentrations of the analyzed inflammatory markers

\begin{tabular}{|c|c|c|c|c|c|c|c|c|}
\hline & \multicolumn{2}{|c|}{ mRNA $L C N 2$} & \multicolumn{2}{|c|}{ mRNA $C H I 3 L 1$} & \multicolumn{2}{|c|}{ mRNA $S P P 1$} & \multicolumn{2}{|c|}{ mRNA RARRES2 } \\
\hline & $r$ & $P$ & $r$ & $P$ & $r$ & $P$ & $r$ & $P$ \\
\hline Age & 0.22 & 0.074 & -0.01 & 0.993 & 0.23 & 0.061 & 0.04 & 0.699 \\
\hline BMI & 0.65 & $<\mathbf{0 . 0 0 1}$ & 0.37 & 0.002 & 0.58 & $<0.001$ & 0.42 & $<\mathbf{0 . 0 0 1}$ \\
\hline Body fat & 0.66 & $<\mathbf{0 . 0 0 1}$ & 0.34 & 0.007 & 0.56 & $<0.001$ & 0.43 & 0.008 \\
\hline Fasting glucose & 0.20 & 0.166 & 0.01 & 0.991 & -0.04 & 0.732 & -0.04 & 0.973 \\
\hline Fasting insulin & 0.48 & $<\mathbf{0 . 0 0 1}$ & 0.29 & 0.036 & 0.27 & 0.055 & 0.24 & 0.097 \\
\hline QUICKI & -0.60 & $<\mathbf{0 . 0 0 1}$ & -0.38 & 0.007 & -0.49 & 0.004 & -0.35 & 0.015 \\
\hline LDL-cholesterol & 0.37 & 0.015 & 0.28 & 0.059 & 0.33 & 0.029 & 0.44 & 0.003 \\
\hline HDL-cholesterol & -0.43 & 0.002 & -0.22 & 0.136 & -0.18 & 0.231 & -0.31 & 0.041 \\
\hline Leptin & 0.51 & 0.003 & 0.27 & 0.057 & 0.45 & 0.002 & 0.36 & 0.015 \\
\hline CRP & 0.53 & $<\mathbf{0 . 0 0 1}$ & 0.27 & 0.071 & 0.43 & 0.003 & 0.10 & 0.523 \\
\hline Fibrinogen & 0.52 & $<\mathbf{0 . 0 0 1}$ & 0.15 & 0.312 & 0.38 & 0.007 & 0.17 & 0.269 \\
\hline von Willebrand factor & 0.27 & 0.078 & 0.29 & 0.048 & 0.39 & 0.008 & 0.28 & 0.066 \\
\hline AST & 0.02 & 0.998 & 0.27 & 0.052 & 0.12 & 0.356 & -0.10 & 0.482 \\
\hline ALT & 0.26 & 0.072 & 0.32 & 0.017 & 0.23 & 0.094 & 0.06 & 0.656 \\
\hline
\end{tabular}

Bold values denote statistically significant $P$ values

$A L T$ alanine aminotransferase, $A S T$ aspartate aminotransferase, $B M I$ body mass index, $C R P$ C-reactive protein, QUICKI quantitative insulin sensitivity check index

\begin{tabular}{|c|c|c|c|c|c|c|c|c|}
\hline & \multicolumn{2}{|c|}{ Circulating LCN-2 } & \multicolumn{2}{|c|}{ Circulating YKL-40 } & \multicolumn{2}{|c|}{ Circulating OPN } & \multicolumn{2}{|c|}{ Circulating chemerin } \\
\hline & $r$ & $P$ & $r$ & $P$ & $r$ & $P$ & $r$ & $P$ \\
\hline mRNA $L C N 2$ & 0.34 & 0.004 & 0.19 & 0.131 & 0.23 & 0.064 & 0.57 & $<\mathbf{0 . 0 0 1}$ \\
\hline mRNA $C H I 3 L 1$ & 0.25 & 0.028 & 0.29 & 0.014 & 0.02 & 0.837 & 0.24 & 0.084 \\
\hline mRNA $S P P 1$ & 0.28 & 0.017 & 0.17 & 0.168 & 0.10 & 0.438 & 0.37 & 0.006 \\
\hline mRNA RARRES2 & 0.24 & 0.048 & 0.08 & 0.510 & 0.20 & 0.103 & 0.42 & $<\mathbf{0 . 0 0 1}$ \\
\hline
\end{tabular}

Bold values denote statistically significant $P$ values response in obesity (Olefsky and Glass 2010). Extensive work has been done on the biology of immune cells that infiltrate the adipose tissue as a major source of pro-inflammatory cytokines, but little is known about the association between PBMC and obesity-associated inflammation. PBMC are a type of cells suitable and of great interest in the research into mechanisms of immune dysfunction in obesity since they are easily accessible and have been also shown to reflect the responses of dietary modifications, oxidative stress and drugs at the gene expression level (de Mello et al. 2012; Ghanim et al. 2004; Yamaoka et al. 2012). In the present study, we show, for the first time, that PBMC gene expression levels of the novel adipokines chemerin, YKL-40, LCN-2 and OPN were strongly upregulated in obesity independently of the glycemic state and associated with different circulating inflammatory markers. Furthermore, the adipokine transcript levels in PBMC from obese subjects reflected their circulating concentrations.

LCN-2, also known as neutrophil gelatinase-associated lipocalin (NGAL) and 24p3, is a component of the innate immune system that also functions as a carrier protein for small hydrophobic molecules including iron (Flo et al. 2004). Upregulated expression levels have been described in both adipose tissue and liver of $d b / d b$ mice and in isolated adipocytes from obese Zucker rats (Zhang et al. 2008). We have recently shown that $\mathrm{LCN}-2$ is highly expressed in visceral adipose tissue in human obesity (Catalán et al. 2009). Accordingly, we detected increased circulating and gene expression levels of $L C N 2$ in human PBMC in obesity as well as a positive association with insulin levels and HOMA index. In this regard, an upregulation of $L C N 2$ by insulin in adipose tissue explants and increased circulating levels after hyperinsulinemic induction in humans have been described (Tan et al. 2009). The positive correlation between both circulating and gene expression levels of LCN-2 with inflammatory markers detected in the present study may be related to the role of LCN-2 as an anti-inflammatory regulator described not only on adipocytes but also on macrophage activation (Guo et al. 2014; Zhang et al. 2008).

OPN regulates a great variety of cellular processes including tissue remodeling and immune regulation through its interactions with multiple cell surface receptors (Kahles 
et al. 2014). Abundant evidence suggests that osteopontin plays a pivotal role in the development of adipose tissue inflammation and insulin resistance (Gómez-Ambrosi et al. 2007; Lancha et al. 2014). This observation is in line with our results, where elevated circulating levels of OPN and its gene expression in PBMC in obesity have been observed. It is well recognized that OPN controls monocyte adhesion and migration and also induces the expression of matrix metalloproteinases proposing a role in regulating monocyte/macrophage recruitment to sites of inflammation (Kahles et al. 2014). Therefore, our findings may also be relevant with respect to migration of PBMC to sites of inflammation such as adipose tissue or liver. OPN deficiency has been described to improve glucose tolerance (Kiefer et al. 2010; Lancha et al. 2014). In this regard, a positive association of mRNA levels of SPP1 in PBMC with insulin resistance has been shown in the present study, suggesting the involvement of PBMC in glucose homeostasis in obesity.

Chemerin is a novel chemo-attractant protein with important roles in the initiation of immune responses by regulating traffic of leukocyte populations. Chemerin has been also shown to be an independent predictor for the number of white blood cells (Landgraf et al. 2012). We clearly showed increased circulating and gene expression levels of this protein in PBMC in both groups of obese patients. Although chemerin promotes inflammation by stimulating macrophage adhesion, an anti-inflammatory effect depending on the protease that cleavages prochemerin has been also reported (Cash et al. 2008). Our findings are in line with previous work in which an association of chemerin with inflammatory factors was detected (Weigert et al. 2010). Several studies suggested chemerin as a predictor of the metabolic syndrome, particularly regarding insulin and glucose homeostasis (Bozaoglu et al. 2007). In the present study, we show a correlation of chemerin with insulin resistance.

YKL-40 mainly functions in tissue inflammation and extracellular matrix remodeling. Our present findings confirm that circulating levels of YKL-40 are increased in obesity (Catalán et al. 2011; Hempen et al. 2009). Moreover, elevated gene expression levels in PBMC in obesity were found indicating the contribution of YKL-40 in the worsening of the inflammatory response in obesity. Interestingly, we detected a positive association of YKL-40 with circulating concentrations of markers of liver function such as AST and ALT. Reportedly, YKL-40 concentrations are increased in patients with chronic liver disease, providing information about the amount of liver fibrosis (Johansen et al. 2000).

In the current study, no differences in circulating and gene expression levels of novel adipokines between NG and T2D obese patients were found, pointing to a comparable inflammatory response in the PBMC of patients from both groups. In agreement with our findings, no differences in the expression of inflammatory genes in adipose tissue or PBMC between metabolically obese healthy and metabolically abnormal obesity groups have been found in recent studies (Catalán et al. 2007a; GómezAmbrosi et al. 2014; Telle-Hansen et al. 2013). Moreover, we have identified that the upregulation of gene expression levels of most of the novel adipokines in PBMC is associated with their circulating concentrations, suggesting the contribution of these types of cells to the increased circulating levels in obesity states.

Cytokines are critical effectors of PBMC function, and their altered expression in obesity contributes to the predisposition to a chronic active inflammatory condition associated with the development of obesity-related comorbidities. The identification and study of novel adipokines involved in the regulation of the delicate balance of interplay between metabolic and immune systems may be useful to clarify the mechanisms induced in obesity. Broadening our knowledge about adipokines will provide new opportunities for developing therapeutic approaches to enhance the capacity of endogenous molecules to prevent stress and inflammatory responses associated with obesity.

Acknowledgments We gratefully acknowledge the valuable collaboration of all the members of the Multidisciplinary Obesity Team (Clínica Universidad de Navarra, Pamplona, Spain). CIBER de Fisiopatología de la Obesidad y Nutrición (CIBERobn) is an initiative of the Instituto de Salud Carlos III (Spain). This work was supported by Fondo de Investigación Sanitaria-FEDER (PI12/00515, PI13/ 00460 and PI14/00950) from the Spanish Instituto de Salud Carlos III, by the Department of Health (48/2011 and 58/2011) of the Gobierno de Navarra of Spain and by Fundación Caja Navarra (20-2014).

\section{Conflict of interest None.}

Ethical standard All procedures followed were in accordance with the ethical standards of the responsible committee on human experimentation (institutional and national) and with the Helsinki Declaration of 1975, as revised in 2000 (5). Informed consent was obtained from all patients for being included in the study. The study was approved, from an ethical and scientific standpoint, by the Hospital's Ethical Committee responsible for research.

\section{References}

Bastard JP et al (2000) Elevated levels of interleukin 6 are reduced in serum and subcutaneous adipose tissue of obese women after weight loss. J Clin Endocrinol Metab 85:3338-3342. doi:10. 1210/jcem.85.9.6839

Bozaoglu K et al (2007) Chemerin is a novel adipokine associated with obesity and metabolic syndrome. Endocrinology 148:4687-4694. doi:10.1210/en.2007-0175

Bustin SA et al (2009) The MIQE guidelines: minimum information for publication of quantitative real-time PCR experiments. Clin Chem 55:611-622. doi:10.1373/clinchem.2008.112797 
Campo A et al (2007) Hyperleptinaemia, respiratory drive and hypercapnic response in obese patients. Eur Respir J 30:223-231. doi:10.1183/09031936.00115006

Cash JL et al (2008) Synthetic chemerin-derived peptides suppress inflammation through ChemR23. J Exp Med 205:767-775. doi:10.1084/jem.20071601

Catalán V et al (2007a) Proinflammatory cytokines in obesity: impact of type 2 diabetes mellitus and gastric bypass. Obes Surg $17: 1464-1474$

Catalán V et al (2007b) Validation of endogenous control genes in human adipose tissue: relevance to obesity and obesity-associated type 2 diabetes mellitus. Horm Metab Res 39:495-500. doi:10.1055/s-2007-982502

Catalán V et al (2009) Increased adipose tissue expression of lipocalin-2 in obesity is related to inflammation and matrix metalloproteinase-2 and metalloproteinase- 9 activities in humans. J Mol Med 87:803-813. doi:10.1007/s00109-009-0486-8

Catalán V et al (2011) Increased circulating and visceral adipose tissue expression levels of YKL-40 in obesity-associated type 2 diabetes are related to inflammation: impact of conventional weight loss and gastric bypass. J Clin Endocrinol Metab 96:200-209. doi:10.1210/jc.2010-0994

Catalán V et al (2013) Increased levels of chemerin and its receptor, chemokine-like receptor-1, in obesity are related to inflammation: tumor necrosis factor- $\alpha$ stimulates mRNA levels of chemerin in visceral adipocytes from obese patients. Surg Obes Relat Dis 9:306-314. doi:10.1016/j.soard.2011.11.001

de Mello VD et al (2008a) Downregulation of genes involved in $\mathrm{NF \kappa B}$ activation in peripheral blood mononuclear cells after weight loss is associated with the improvement of insulin sensitivity in individuals with the metabolic syndrome: the GENOBIN study. Diabetologia 51:2060-2067. doi:10.1007/ s00125-008-1132-7

de Mello VD et al (2008b) Effect of weight loss on cytokine messenger RNA expression in peripheral blood mononuclear cells of obese subjects with the metabolic syndrome. Metabolism 57:192-199. doi:10.1016/j.metabol.2007.08.024

de Mello VD, Kolehmanien M, Schwab U, Pulkkinen L, Uusitupa M (2012) Gene expression of peripheral blood mononuclear cells as a tool in dietary intervention studies: what do we know so far? Mol Nutr Food Res 56:1160-1172. doi:10.1002/mnfr.201100685

Diagnosis and classification of diabetes mellitus (2014) Diabetes Care 37(Suppl 1):S81-S90. doi:10.2337/dc14-S081

Dicker D, Salook MA, Marcoviciu D, Djaldetti M, Bessler H (2013) Role of peripheral blood mononuclear cells in the predisposition of obese individuals to inflammation and infection. Obes Facts 6:146-151. doi:10.1159/000350775

Dixon JB, O'Brien PE (2006) Obesity and the white blood cell count: changes with sustained weight loss. Obes Surg 16:251-257. doi:10.1381/096089206776116453

Esser N, Legrand-Poels S, Piette J, Scheen AJ, Paquot N (2014) Inflammation as a link between obesity, metabolic syndrome and type 2 diabetes. Diabetes Res Clin Pract 105:141-150. doi:10. 1016/j.diabres.2014.04.006

Feuerer M et al (2009) Lean, but not obese, fat is enriched for a unique population of regulatory $\mathrm{T}$ cells that affect metabolic parameters. Nat Med 15:930-939. doi:10.1038/nm.2002

Flo TH et al (2004) Lipocalin 2 mediates an innate immune response to bacterial infection by sequestrating iron. Nature 432:917-921. doi:10.1038/nature03104

Fogeda M, Gallart L, Gutiérrez C, Vendrell J, Simón I, García-España A, Richart C (2004) High expression of tumor necrosis factor $\alpha$ receptors in peripheral blood mononuclear cells of obese type 2 diabetic women. Eur Cytokine Netw 15:60-66

Frühbeck G (2012) Obesity: screening for the evident in obesity. Nat Rev Endocrinol 8:570-572. doi:10.1038/nrendo.2012.165
Frühbeck G, Gómez-Ambrosi J (2003) Control of body weight: a physiologic and transgenic perspective. Diabetologia 46:143-172. doi:10.1007/s00125-003-1053-4

Frühbeck G, Toplak H, Woodward E, Yumuk V, Maislos M, Oppert JM (2013) Obesity: the gateway to ill health-an EASO position statement on a rising public health, clinical and scientific challenge in Europe. Obes Facts 6:117-120. doi:10.1159/ 000350627

Ghanim H, Aljada A, Hofmeyer D, Syed T, Mohanty P, Dandona P (2004) Circulating mononuclear cells in the obese are in a proinflammatory state. Circulation 110:1564-1571. doi:10.1161/ 01.CIR.0000142055.53122.FA

Gómez-Ambrosi J et al (2004) Gene expression profile of omental adipose tissue in human obesity. FASEB J 18:215-217. doi:10. 1096/fj.03-0591fje

Gómez-Ambrosi J et al (2006) Increased serum amyloid A concentrations in morbid obesity decrease after gastric bypass. Obes Surg 16:262-269. doi:10.1381/096089206776116525

Gómez-Ambrosi J et al (2007) Plasma osteopontin levels and expression in adipose tissue are increased in obesity. J Clin Endocrinol Metab 92:3719-3727. doi:10.1210/jc.2007-0349

Gómez-Ambrosi J et al (2012) Body mass index classification misses subjects with increased cardiometabolic risk factors related to elevated adiposity. Int J Obes 36:286-294. doi:10.1038/ijo.2011. 100

Gómez-Ambrosi J et al (2014) Increased cardiometabolic risk factors and inflammation in adipose tissue in obese subjects classified as metabolically healthy. Diabetes Care 37:2813-2821. doi:10. 2337/dc14-0937

Guo H, Jin D, Chen X (2014) Lipocalin 2 is a regulator of macrophage polarization and NF-kappaB/STAT3 pathway activation. Mol Endocrinol 28:1616-1628. doi:10.1210/me.2014-1092

Hempen $\mathrm{M}$ et al (2009) YKL-40 is elevated in morbidly obese patients and declines after weight loss. Obes Surg 19:1557-1563. doi:10.1007/s11695-009-9917-4

Johansen JS, Christoffersen P, Moller S, Price PA, Henriksen JH, Garbarsch C, Bendtsen F (2000) Serum YKL-40 is increased in patients with hepatic fibrosis. J Hepatol 32:911-920

Johnson AM, Olefsky JM (2013) The origins and drivers of insulin resistance. Cell 152:673-684. doi:10.1016/j.cell.2013.01.041

Kahles F, Findeisen HM, Bruemmer D (2014) Osteopontin: a novel regulator at the cross roads of inflammation, obesity and diabetes. Mol Metab 3:384-393. doi:10.1016/j.molmet.2014.03. 004

Kiefer FW et al (2010) Neutralization of osteopontin inhibits obesityinduced inflammation and insulin resistance. Diabetes 59:935-946. doi:10.2337/db09-0404

Klöting N, Blüher M (2014) Adipocyte dysfunction, inflammation and metabolic syndrome. Rev Endocr Metab Disord 15:277-287. doi:10.1007/s11154-014-9301-0

Lancha A et al (2014) Osteopontin deletion prevents the development of obesity and hepatic steatosis via impaired adipose tissue matrix remodeling and reduced inflammation and fibrosis in adipose tissue and liver in mice. PLoS One 9:e98398. doi:10. 1371/journal.pone.0098398

Landgraf $\mathrm{K}$ et al (2012) Chemerin as a mediator between obesity and vascular inflammation in children. J Clin Endocrinol Metab 97:E556-E564. doi:10.1210/jc.2011-2937

Lasselin J et al (2014) Adipose inflammation in obesity: relationship with circulating levels of inflammatory markers and association with surgery-induced weight loss. J Clin Endocrinol Metab 99:E53-E61. doi:10.1210/jc.2013-2673

Leal Vde O, Mafra D (2013) Adipokines in obesity. Clin Chim Acta 419:87-94. doi:10.1016/j.cca.2013.02.003

Lee CT, Harris SB, Retnakaran R, Gerstein HC, Perkins BA, Zinman B, Hanley AJ (2014) White blood cell subtypes, insulin 
resistance and $\beta$-cell dysfunction in high-risk individuals-the PROMISE cohort. Clin Endocrinol 81:536-541. doi:10.1111/ cen. 12390

Lumeng CN, Saltiel AR (2011) Inflammatory links between obesity and metabolic disease. J Clin Invest 121:2111-2117. doi:10. 1172/JCI57132

Lumeng CN, Bodzin JL, Saltiel AR (2007) Obesity induces a phenotypic switch in adipose tissue macrophage polarization. J Clin Invest 117:175-184. doi:10.1172/JCI29881

$\mathrm{Ng} \mathrm{M}$ et al (2014) Global, regional, and national prevalence of overweight and obesity in children and adults during 1980-2013: a systematic analysis for the Global Burden of Disease Study 2013. Lancet 384:766-781. doi:10.1016/S0140-6736(14)60460-8

Olefsky JM, Glass CK (2010) Macrophages, inflammation, and insulin resistance. Annu Rev Physiol 72:219-246. doi:10.1146/ annurev-physiol-021909-135846

O'Rourke RW et al (2006) Alterations in peripheral blood lymphocyte cytokine expression in obesity. Clin Exp Immunol 146:39-46. doi:10.1111/j.1365-2249.2006.03186.x

Ouchi N, Parker JL, Lugus JJ, Walsh K (2011) Adipokines in inflammation and metabolic disease. Nat Rev Immunol 11:85-97. doi:10.1038/nri2921

Poulain-Godefroy O, Lecoeur C, Pattou F, Fruhbeck G, Froguel P (2008) Inflammation is associated with a decrease of lipogenic factors in omental fat in women. Am J Physiol Reg Int Comp Physiol 295:R1-R7. doi:10.1152/ajpregu.00926.2007

Sakaguchi S, Yamaguchi T, Nomura T, Ono M (2008) Regulatory T cells and immune tolerance. Cell 133:775-787. doi:10.1016/j. cell.2008.05.009

Tan BK, Adya R, Shan X, Syed F, Lewandowski KC, O'Hare JP, Randeva HS (2009) Ex vivo and in vivo regulation of lipocalin2, a novel adipokine, by insulin. Diabetes Care 32:129-131. doi: $10.2337 / \mathrm{dc} 08-1236$

Telle-Hansen VH et al (2013) Altered expression of genes involved in lipid metabolism in obese subjects with unfavourable phenotype. Genes Nutr 8:425-434. doi:10.1007/s12263-012-0329-Z
Vozarova B, Weyer C, Lindsay RS, Pratley RE, Bogardus C, Tataranni PA (2002) High white blood cell count is associated with a worsening of insulin sensitivity and predicts the development of type 2 diabetes. Diabetes 51:455-461

Wagner NM et al (2013) Circulating regulatory $\mathrm{T}$ cells are reduced in obesity and may identify subjects at increased metabolic and cardiovascular risk. Obesity 21:461-468. doi:10.1002/oby.20087

Weigert $\mathbf{J}$ et al (2010) Systemic chemerin is related to inflammation rather than obesity in type 2 diabetes. Clin Endocrinol 72:342-348. doi:10.1111/j.1365-2265.2009.03664.x

Weisberg SP, McCann D, Desai M, Rosenbaum M, Leibel RL, Ferrante AW Jr (2003) Obesity is associated with macrophage accumulation in adipose tissue. J Clin Invest 112:1796-1808. doi:10.1172/JCI19246

$\mathrm{Xu} \mathrm{H}$ et al (2003) Chronic inflammation in fat plays a crucial role in the development of obesity-related insulin resistance. J Clin Invest 112:1821-1830. doi:10.1172/JCI19451

Yamaoka M et al (2012) A pilot investigation of visceral fat adiposity and gene expression profile in peripheral blood cells. PLoS One 7:e47377. doi:10.1371/journal.pone.0047377

Yamaoka M et al (2013) Gene expression levels of S100 protein family in blood cells are associated with insulin resistance and inflammation (peripheral blood S100 mRNAs and metabolic syndrome). Biochem Biophys Res Commun 433:450-455. doi:10.1016/j.bbrc.2013.02.096

Yudkin JS, Stehouwer CD, Emeis JJ, Coppack SW (1999) C-reactive protein in healthy subjects: associations with obesity, insulin resistance, and endothelial dysfunction: a potential role for cytokines originating from adipose tissue? Arterioscler Thromb Vasc Biol 19:972-978

Zhang J, Wu Y, Zhang Y, Leroith D, Bernlohr DA, Chen X (2008) The role of lipocalin 2 in the regulation of inflammation in adipocytes and macrophages. Mol Endocrinol 22:1416-1426. doi:10.1210/me.2007-0420 